\title{
Categorical time production: Evidence for discrete timing in motor control
}

\author{
CHARLES E. COLLYER \\ University of Rhode Island, Kingston, Rhode Island \\ and \\ HILARY A. BROADBENT and RUSSELL M. CHURCH \\ Brown University, Providence, Rhode Island
}

\begin{abstract}
Subjects performed a repetitive manual tapping task, attempting to match a given rate of auditory stimulus pulses, first with the pulses audible (synchronization) and then with the pulses turned off (continuation). In different sessions, the interstimulus interval (ISI) was selected from the range 175 to $825 \mathrm{msec}$ in steps of $25 \mathrm{msec}$, with different ISI values presented in a random order. Across this range of ISI conditions, interresponse intervals (IRIs) exhibited alternating positive bias (too slow) and negative bias (too fast). We interpret this pattern of bias in terms of a discrete, or categorical, timing mechanism in motor timing. Categorical time production can be viewed as extending our conception of the timekeeper in Wing's (Wing \& Kristofferson, 1973a, 1973b) two-process model of motor timing and may be related to the system of multiple clocks proposed by Kristofferson (1980) to explain a categorical pattern of variability measures in duration discrimination.
\end{abstract}

Should we conceive of psychological time as a smooth flow or as a succession of discrete moments? Introspection seems to favor smoothness. William James (1890/1950, p. 237) described consciousness as "sensibly continuous," meaning that the flow of time as we experience it is not noticeably punctuated by regular markers of its passage. In discussing the perception of time itself, James emphasized its subdivision by external events rather than internal rhythms. In motor behavior, as well as in perception, timing seems to be continuously adjustable, within broad limits. For example, when we speed up or slow down in pedaling a bicycle, or adjust to different musical tempos in dancing or playing an instrument, it seems about equally easy to meet timing demands over a range of rates. Introspection thus suggests that psychological time is continuous rather than discrete. If we have internal clocks helping us to perform these and other acts of timing, they tick unobtrusively and seem capable of many rate gradations.

However, subjective experience may not be the best indicator of the mechanisms responsible for timing. Even if, because of some neurophysiological necessity, timing is grounded in the periodic marking off of discrete moments, the perceptual and motor needs of organisms might be better served by a close approximation to "sensibly continuous" time than by noticeably quantized time. A

Preparation of this article was aided by Grant NSS2-S07-RR07086-14 to the University of Rhode Island from the National Center for Research Resources. The experiment reported here was presented at the 31st Annual Meeting of the Psychonomic Society, New Orleans, November 1990. The authors are grateful to L. G. Allan and D. A. Rosenbaum for their constructive criticism of an earlier draft. Address correspondence to the first author at the Department of Psychology, URI, Kingston, RI 02881-0808. suggestive comparison can be made with the subjective smoothness of visual scanning, despite the discreteness of saccadic eye movements (Matin, 1974).

\section{Discrete Versus Continuous Timing}

In this study, we entertained the hypothesis of an underlying discreteness of timing in the control of repetitive manual tapping movements. We used a well-known task in which the subjects first tap in synchrony with periodic auditory pulses (the synchronization phase of a trial) and then continue tapping at the same rate but without the auditory pulses (the continuation phase of a trial). Figure 1 shows the basic features of such a trial and indicates the meaning of two terms we shall use in describing the experiment: the interstimulus interval (ISI) and the interresponse interval (IRI).

This type of tapping task has been used by many researchers (e.g., Bartlett \& Bartlett, 1959; Dunlap, 1910; Franek, Radil, Indra, \& Lansky, 1987; Hary \& Moore, 1985, 1987; Nagasaki \& Nagasaki, 1982; Peters, 1989; Stevens, 1886; Wing, 1980; Wing, Church, \& Gentner, 1989; Woodrow, 1932) to investigate various aspects of motor timing. Tapping in synchrony with a pacer stimulus demands the coordination of exogenous with endogenous timing, whereas tapping in continuation must rely on endogenous timing. Thus, we would expect continuation data to give the clearest picture of an endogenous timing mechanism.

\section{Continuation}

Figure 2 shows how continuous and discrete timing processes might be reflected in the IRIs of the continuation phase. 


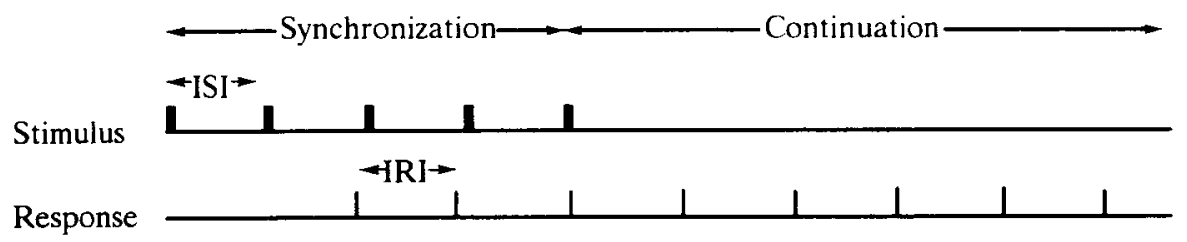

Figure 1. Synchronization and continuation phases of one trial. Each phase consisted of 50 taps. ISI is the interstimulus interval of auditory pacer sounds; IRI is the interresponse interval of taps.

In each panel on the left side of Figure 2, the abscissa is ISI, defined by the time in milliseconds ( $\mathrm{msec}$ ) between onsets of successive auditory pulses during the synchronization phase of a trial. ISI sets the interval that the subject's tapping is intended to match during the continuation phase. The ordinate represents the actual rate of tapping during continuation, expressed as IRI in msec. (In our analyses, we used median IRI for each tapping trial and the mean of medians over trials.) Each panel on the right side of Figure 2 converts IRI to a bias measure, defined as the difference between IRI and ISI.

We use the term bias to refer to systematic inaccuracy of time production in the tapping task. Three types of bias are defined as follows:

$$
\text { Raw Bias = IRI - ISI. }
$$

Raw bias measures the departure of time production from the intended value defined by ISI.

$$
\text { Linear Bias = Pred(IRI) - ISI, }
$$

where Pred(IRI) is the predicted value of IRI from simple linear regression, with ISI as the predictor. Linear bias measures the departure at a particular ISI of the regression line from the identity function. The set of linear bias values for a set of data can be derived from the regression slope and intercept.

$$
\text { Residual Bias = IRI - Pred(IRI). }
$$

Residual bias measures the departure of time production from the value predicted by simple linear regression. We will see that the question of continuous versus categorical timing is related to whether the relationship between residual bias and ISI is random or exhibits meaningful structure.

These definitions imply that linear bias and residual bias constitute a partitioning of raw bias:

$$
\text { Raw Bias = Linear Bias + Residual Bias. }
$$

In Figure 2, linear bias was assumed to be zero at all values of ISI; hence, raw bias can be equated with residual bias.

The two panels at the top of Figure 2 represent continuously adjustable tapping. IRI is a linear function of ISI, and, in the absence of linear bias, it is the identity function. Any residual bias is expected to be randomly dispersed around zero at all values of ISI. More generally, at each value of ISI, we would expect the residual bias to be randomly dispersed around the value of IRI predicted by linear regression.
In contrast, the middle two panels of Figure 2 represent motor timing that is discrete and inflexible. Tapping is only possible at the two IRIs labeled $\mathrm{C} 1$ and $\mathrm{C} 2$. These two intervals might reflect either the available rates of an internal clock or the possible output rates of the motor system. As a general term, we will refer to them as critical intervals. Under some theories, critical intervals could be related, one being double the other, for example. The function relating IRI and ISI under this extreme degree of discreteness is a step function, as shown in the middleleft panel. The middle-right panel shows the expected pat-
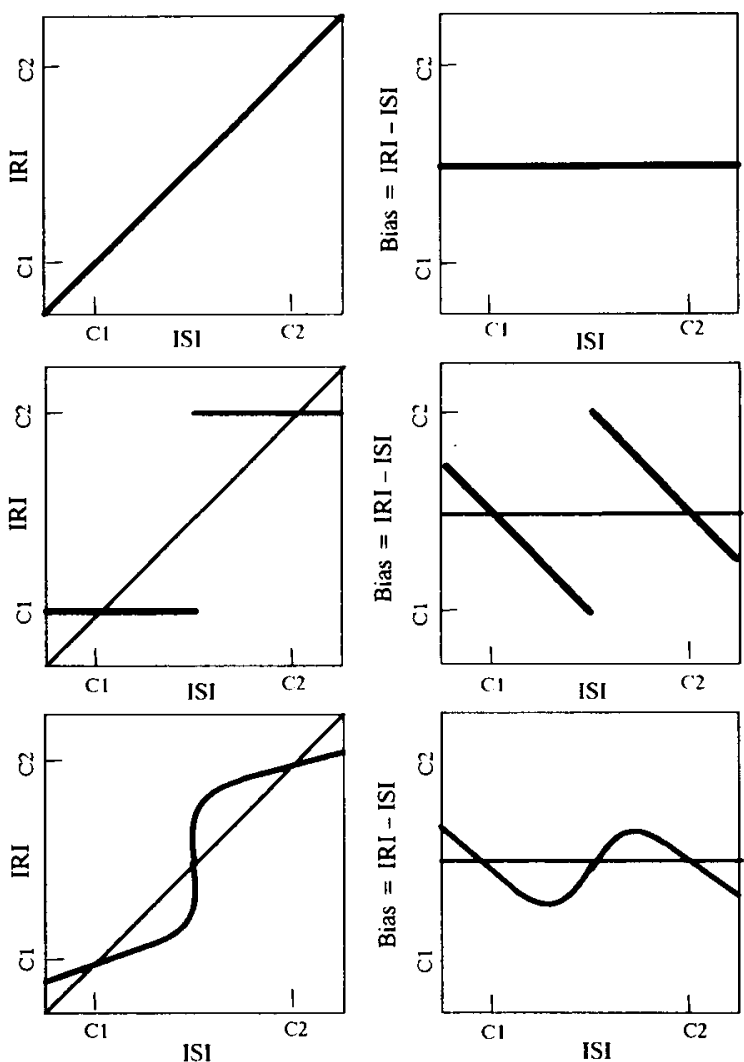

Figure 2. Functions relating Interresponse interval (IRI) and interstimulus interval (ISI) (lent) and corresponding patterns of blas (right) under three conceptions of motor timing. Top: Continuously adjustable, unbiased timing. Middle: Categorical timing with inflexible oscillators. Bottom: Categorical timing with partial entrainment or mixture of oscillators. 
tern of raw and residual bias for this case, which, as in the case illustrated in the top two panels, excludes linear bias. Notice that tapping is relatively slow (positive bias) at ISIs shorter than the critical intervals and relatively fast (negative bias) at ISIs longer than these intervals. We will refer to such an ordered pattern of bias as an oscillator signature. In the more general case in which linear bias is present, the oscillator signature would alternate around the overall regression line.

The bottom panels of Figure 2 represent an intermediate case. Performance can be viewed as the output of a mechanism that is basically discrete but flexible enough to approximate continuous timing. (For example, mechanisms involving partial adjustability of local clocks, and/or a mixture of the influences of several clocks, are possible.) The observed IRI would be a weighted average of the ISI, linear bias, the local bias determined by the underlying oscillatory mechanism, and any random components of performance. The function relating bias and ISI could have a complicated form, depending on the local influence of these factors and on whether or not critical intervals such as $\mathrm{C} 1$ and $\mathrm{C} 2$ have overlapping ranges of influence. In Figure 2, we have suggested a function form consisting of alternating subranges of relatively slow and relatively fast tapping, which may be understood as a generalization of the discrete oscillator signature.

The import of Figure 2 is that categorical timing should be reflected in nonlinearities of the IRI and bias functions, particularly in higher order nonlinearities. The advantage of looking at residual bias is that, with linear dependence on ISI and linear bias removed, one focuses on the question of whether the data exhibit further nonlinear dependence on ISI (supporting a categorical account) or independence (supporting a continuity account).

\section{Synchronization}

IRIs in synchronization should be highly accurate, on the average, because the presence of the exogenous pacer stimuli provides a basis for correction of the tapping rate. Therefore, we would not expect to see much linear bias in synchronization and would expect any oscillatory residual bias to be less than in continuation. The stimulus-response interval (rather than IRI) would probably be the appropriate measure for testing theories of synchronization (e.g., Hary \& Moore, 1985, 1987). However, in the present paper, we will focus on the analysis of IRI in continuation.

\section{Variability}

In many types of timing performance, longer time intervals are associated with increased variability. Workers in both time perception (e.g., Church, Getty, \& Lerner, 1976) and time production (e.g., Wing, 1980; Wing, Church, \& Gentner, 1989) have asked whether the standard deviation or the variance of psychological time is linearly related to real time. Scalar models propose that the standard deviation increases linearly with time (Gibbon, $1977,1991)$. Thus, a way of testing for the scalar property in tapping data would be to regress the standard deviation of IRI against ISI. On the other hand, some models predict that the variance, rather than the standard deviation, increases linearly with time-for example, if a long interval is composed of shorter component intervals (Creelman, 1962).

After allowing for an overall increase in variability with ISI, the variability of the IRIs might also show alternating increases and decreases across neighboring ISIs. For example, subjects could find it easy to maintain a rate of tapping when the ISI is close to one of their critical intervals but require frequent corrections of timing drift when trying to match more remote rates. If so, we would expect low variability of IRIs in ISI conditions corresponding to critical intervals and higher variability in other conditions. On the other hand, the critical intervals may be those for which timing is most tolerant of variability, so that ISI conditions corresponding to critical intervals would yield higher rather than lower variability in IRI. Color vision provides an analogy in which wavelengths are discriminated better (exhibit lower performance variability) near category boundaries than in the middle of color categories. Sharper discrimination at category boundaries also characterizes other types of categorical perception (Harnad, 1987).

\section{Central Timing or Motor-System Constraints?}

There are at least two possible interpretations of the critical intervals $\mathrm{C1}$ and $\mathrm{C2}$ in Figure 2. One possibility is that $\mathrm{C} 1$ and $\mathrm{C} 2$ represent the natural periods of a centralpattern generator (Glass \& Mackey, 1988) or other neural timekeeping mechanism; the other possibility is that such intervals emerge from biomechanical constraints and are unrelated to timekeeping functions. Thus, finding a nonlinear function relating IRI to ISI would not, in itself, indicate discreteness of central timing. However, if it could be shown that such a function was invariant across different biomechanical conditions, the case for a timing interpretation would be strengthened. Two biomechanical conditions that have been shown to be different enough to produce other motor timing effects (Wing, 1977; Rosenbaum, Slotta, Vaughan, \& Plamondon, 1991) were compared in a recent study of preferred rates of tapping (Collyer, Broadbent, \& Church, 1990). The results of this study, summarized below, suggest that these conditions are reasonable candidates for demonstrating invariance of an oscillator signature across conditions differing in the physical details of response execution.

Collyer et al. (1990) studied subjectively preferred rates of manual tapping under two biomechanically different conditions. In one condition, subjects tapped by moving their index finger, with the wrist restrained by a brace; in the other condition, subjects tapped by moving their wrist, with the fingers restrained by a bandage. Preferred rates of tapping were very similar in the finger and wrist conditions, although individual subjects differed considerably from one another. Because the finger and wrist conditions did not differ, we concluded that the dynamics of the motor system, at the level of these effectors, do not 
strongly determine preferred rate. Instead, either of these effectors may be used to implement a rate that is apparently determined by some other part of the system. The origin of preferred rates could be at a higher level of the motor system, imposing constraints on both wrist and finger, or it could be in a central timing process.

The preferred-rate study indicated that the biomechanical manipulation did not change the rates that were "comfortable" for subjects to produce. However, this result leaves open the question of how biomechanical constraints affect time production when the subject's task is to match an externally specified rate. Therefore, the present study also included a comparison of finger and wrist tapping for some of the subjects.

\section{METHOD}

\section{Subjects}

Five subjects participated in the experiment. All were predominantly right-handed. Two subjects (A.S. and J.W.) were graduate students in the Brown University Department of Psychology who were unfamiliar with the hypotheses under investigation. The other subjects (C.C., H.B., and R.C.) were the authors.

\section{Apparatus}

The experiment was controlled by an IBM AT computer. Stimuli and responses were timed by a MetraByte CTM05 I/O board. Auditory stimuli (50-msec, 1-kHz tones) were generated by a MED Associates signal generator (ANL-916). The subjects heard the tones over headphones (Realistic Nova 40). Tapping responses were made on a fixed metallic contact plate (a U.S. penny). The subject's forearm rested on a sheet of foil through which a weak current was passed; touching the contact plate closed a circuit, signaling a tap to the computer. A software debouncing routine filtered the record of responses by requiring a minimum of $75 \mathrm{msec}$ between successive taps. This criterion successfully discriminated between the short intervals (usually 5-15 msec) occasionally produced when finger contact was intermittently broken at the beginning or end of a tap and the longer intervals (usually greater than $100 \mathrm{msec}$, even at fast rates of tapping) produced between deliberate taps.

\section{Design and Procedure}

A trial consisted of 50 synchronization taps and 50 continuation taps. During the synchronization phase of a trial, a train of 50-msec auditory tones (pacer sounds) was heard over the headphones, and the subject's task was to tap in synchrony with these sounds. After 50 synchronization taps, the computer terminated the pacer sounds and went on to record the 50 continuation taps. The subject's task was to maintain a rate of tapping equal to the rate of the pacer sounds throughout both the synchronization and continuation phases of the trial. The end of a trial was signaled by a distinctive sound (two short tones).

A block of five consecutive trials was completed at each ISI. A session consisted of from three to six blocks. Generally, one session was done each day, for several days, until a replication of the 27 ISIs from 175 to $825 \mathrm{msec}$ (in steps of $25 \mathrm{msec}$ ) had been completed. Each replication was carried out under either the wrist (W) or finger $(F)$ condition, as described below. The ISI block sequence was random, and different for each subject and condition.

The subjects were tested individually while seated at a table and wearing headphones. Tapping was done with the right hand. In both wrist and finger conditions, the forearm rested on a foil-covered surface about $35-40 \mathrm{~cm}$ from the contact plate. In the wrist condition, finger movements were restrained by an Ace bandage wrapped around the hand, with the index finger extended and the other fingers folded. The hand was then relatively rigid but able to move freely at the wrist. In the finger condition, wrist movements were restrained by an orthopedic wrist brace (Scott Specialties Inc., No. 4039), which left the index finger free to move.

Subjects C.C. and H.B. each completed a replication of the finger condition (denoted by Old[F]) by using an early version of the experimental software that provided some but not all of the measures available with the final version. As a result of using the later software, the ordering of conditions for each subject was as follows: C.C.-W, F; H.B.-F, W; R.C.-F, W; A.S.-F only; J.W.-F only.

Each trial yielded a distribution of 49 continuation IRIs, from which the median IRI was computed. The mean of the five trial medians at each ISI was the measure of IRI subjected to analysis. However, in two data sets (denoted by C.C.-Old[F] and H.B.-Old[F]) obtained with the earlier version of the experimental software, the mean of the 245 continuation IRIs at each ISI was used.

We chose to use the median to measure central tendency because it is unaffected by occasional outliers. When outliers occurred, they consisted of IRIs that were about double the typical value, and could be attributed to missed taps. In the data presented here, the median was very close to the mean for all trials without such outliers, indicating symmetry of the underlying IRI distributions. We adopted the semi-interquartile range as our measure of variability in preference to the standard deviation, again because it is less sensitive to outliers. (For symmetrical distributions, the semi-interquartile range is proportional to the standard deviation, and its square is proportional to variance.)

\section{RESULTS AND DISCUSSION}

Simple regression was used to fit the IRI versus ISI function for each data set. Table 1 gives the slope, intercept, and proportion of variance accounted for $\left(r^{2}\right)$ for each fitted line. Even the data set giving the poorest linear fit, that of subject R.C. in the finger condition (R.C. -F), conformed closely to a linear function, with $r^{2}=.996$. It is clear that at this level of description, performance is well described by a simple linear model.

There were small departures from an identity relationship (i.e., slope of 1 and intercept of 0 ) between IRI and ISI, as indicated in Table 1. Such departures define the linear bias of a data set and are consistent with continuity of timing. Residual bias was defined as the difference be-

Table 1

Regression Coefiicients for IRI as a Function of ISI

\begin{tabular}{lccc}
\hline \multicolumn{1}{c}{ Data Set } & Slope & $\begin{array}{c}\text { Intercept } \\
\text { (in msec) }\end{array}$ & $\boldsymbol{r}^{2}$ \\
\hline C.C.-F & $0.96 \dagger$ & 8.9 & .998 \\
C.C.-W & $0.96 \dagger$ & 5.6 & .997 \\
C.C.-Old(F) $\ddagger$ & $0.97 \dagger$ & 10.7 & .998 \\
H.B.-F & $1.02^{*}$ & -10.7 & .997 \\
H.B.-W & $1.05 \dagger$ & $-20.8 \dagger$ & .996 \\
H.B.-Old(F) $\ddagger$ & 1.00 & 2.8 & .997 \\
R.C.-F & 0.99 & 7.1 & .996 \\
R.C.-W & 1.01 & -2.5 & .997 \\
A.S.-F & $1.05 \dagger$ & $-13.2 \dagger$ & .997 \\
J.W.-F & 0.98 & -4.2 & .997 \\
\hline
\end{tabular}

Note-The tests reported here were for the null hypotheses slope = 1.0 and intercept $=0$; all slopes were significantly different from zero. ${ }^{*} p<.05$. ${ }^{\dagger} p<.01$. $¥$ For the data sets C.C. - Old(F) and H.B.-Old(F), mean IRI was used as the criterion variable rather than the mean of the five trial medians. 
Table 2

Regression Coefficients for the Semi-Interquartile Range of IRI and Its Square as Functions of ISI

\begin{tabular}{|c|c|c|c|c|c|c|}
\hline \multirow[b]{2}{*}{ Data Set } & \multicolumn{3}{|c|}{ SIQR } & \multicolumn{3}{|c|}{ SIQR $^{2}$} \\
\hline & Slope* & Intercept & $r^{2}$ & Slope* & Intercept & $r^{2}$ \\
\hline C.C. $-F$ & .016 & $3.7^{*}$ & .831 & .373 & -31.9 & .816 \\
\hline C.C. $-\mathrm{W}$ & .020 & $2.6^{*}$ & .868 & .501 & $-71.5^{*}$ & .819 \\
\hline C.C. - Old(F) $\dagger$ & .025 & $5.2^{*}$ & .894 & .880 & -92.1 & .889 \\
\hline H.B. $-F$ & .013 & $6.1^{*}$ & .764 & .341 & 9.7 & .744 \\
\hline H.B. -W & .009 & $7.8^{*}$ & .374 & .229 & 50.3 & .346 \\
\hline H.B.-Old(F) $\dagger$ & .016 & $10.7^{*}$ & .464 & .630 & 68.9 & .406 \\
\hline R.C. $-\mathrm{F}$ & .020 & 2.0 & .846 & .557 & $-105.2^{*}$ & .786 \\
\hline R.C. -W & .022 & 0.3 & .850 & .599 & $-137.6^{*}$ & .608 \\
\hline A.S.-F & .038 & 2.2 & .789 & 1.835 & $-374.3^{*}$ & .709 \\
\hline J.W. $-\mathrm{F}$ & .026 & 2.6 & .854 & .861 & $-149.2^{*}$ & .809 \\
\hline
\end{tabular}

Note-SIQR ${ }^{2}$ in this analysis was the mean of the five-trial values of SIQR ${ }^{2}$, not the square of the mean SIQR. * Significantly different from zero $(p<.01)$. All slopes were significant. †For the data sets C.C.-Old(F) and H.B.-Old(F), the standard deviation and variance were used as the two criterion measures, rather than SIQR and its square.

tween observed IRI and the predicted IRI generated by the fits of Table 1 . The overall pattern of variability of IRI suggested that residual bias be transformed to percent; a rationale for this transformation follows.

In general, the semi-interquartile range (SIQR) of IRI was linearly related to ISI, as indicated by the statistically significant slope values of Table 2. (For the two older data sets, the standard deviation, which can be assumed proportional to SIQR, was used.) The square of SIQR also increased with ISI, but the linear relationship was less strong for all subjects and conditions. Table 2 thus confirms that tapping data exhibit the scalar property, which is a ubiquitous feature of timing performance in perceptual and cognitive tasks (Gibbon, Church, \& Meck, 1984). Given the scalar property, it is appropriate to use percentages to express bias in an equivalent way across different rates of tapping. Percent residual bias (PRB) was defined as follows:

$$
\text { PRB }=\frac{\text { IRI }- \text { Pred(IRI) }}{\text { Pred(IRI) }} \times 100 .
$$

That is, residual bias was expressed as a percentage of the IRI predicted from regression.

\section{An Example of the Pattern of Raw Biases}

The top panel of Figure 3 shows mean IRI as a function of ISI for the data set C.C.-Old(F). The overall linearity of this function is apparent both from the regression $r^{2}$ value of .998 (see Table 1) and from visual inspection of the graph. The bottom panel of Figure 3 shows the pattern of raw biases obtained by subtracting ISI from each mean IRI. The band around zero bias represents a 99\% confidence interval at each ISI for the null hypothesis that IRI equals ISI, based on an assumption of normality. (Mean and median IRIs were very similar across trials at each ISI, implying approximate symmetry of the trial IRI distributions. With $N=245$ continuation IRIs per ISI, the width of the $99 \%$ confidence interval is approximately one-third of the standard deviation of IRI [i.e., $\pm 2.58(S D) / \sqrt{245}=.33(S D)]$. Figure 3 indicates that although IRI is close to being a linear function of ISI, its small departures from ISI are not random. Raw bias differs significantly from zero more often than would be expected by chance because of two features of the data: First, raw bias tends overall to decrease as ISI increases (i.e., there is linear bias); second, raw bias also exhibits local increases and decreases not captured by an overall
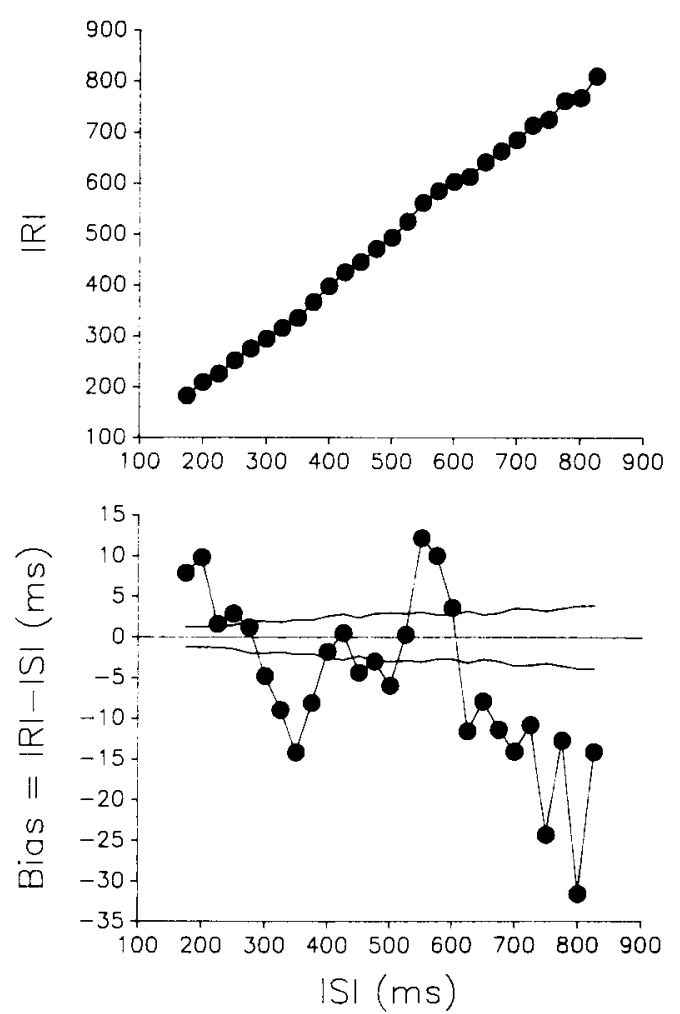

Figure 3. Top: Mean interresponse interval (IRI) as a function of interstimulus interval (ISI) in data set C.C. -Old(F). Bottom: Pattern of raw bias in data set C.C. -Old(F). Each data point represents the difference between mean IRI ( $N=245$ taps, collected over five trials) and ISI. The Gaussian $99 \%$ confidence band, centered on zero bias, is also shown. We use the term osclllator stinature for the alternating pattern of bias illustrated here. 
linear trend (i.e., there is nonlinear structure in the residual biases, an oscillator signature).

\section{A Note on Transformations}

The raw bias values shown in Figure 3 are more often negative than positive and are more variable at large ISIs. Transforming the data to residual bias tended to equate the incidence of positive and negative bias values. Further transforming to PRB provided a correction for the scalar property and so tended to equate the ranges of values found at different ISIs. Since subsequent figures use PRB, the bias functions will look somewhat more symmetrical than the one in Figure 3.

\section{Patterns of Percent Residual Bias (PRB)}

A one-way analysis of variance was performed on the 135 values of PRB ( 27 ISI conditions $\times 5$ trials per condition) of each data set to determine if the conditions differed on this measure. For all 10 data sets, conditions differed significantly: $F(26,108)$ ranged from 3.6 to 24.6 , $p<.001$, and the corresponding multiple $r$ values ranged from .68 to .93 . Under continuity of timing, one would expect no differences among the conditions after the removal of linear structure; however, differences seem to be the rule. Trend analyses were carried out on each data set, with varied results. Quadratic, cubic, and quartic trends were found in different combinations, as shown in Table 3. The trends thus did not provide one standard pattern of differences in PRB across ISI conditions. What, then, was the nature of these differences?

Figure 4 shows PRB as a function of ISI for the 3 subjects who provided more than one replication of the full set of ISIs. All 3 of these subjects performed in the wrist and finger conditions. Figure 4 also includes data from the C.C.-Old(F) and H.B.-Old(F) replications.

Three features of these functions are notable. First, each subject showed alternation of positive and negative residual bias across ISIs, which is the pattern we have

Table 3

Summary of Nonlinear Trends in Percent Residual Bias

\begin{tabular}{lccc}
\hline & Quadratic & Cubic & Quartic \\
\hline C.C.-F & & $*$ & $*$ \\
C.C.-W & $*$ & & $*$ \\
C.C.-Old(F) & & $\dagger$ & $\dagger$ \\
H.B.-F & $\dagger$ & & \\
H.B.-W & $\dagger$ & & \\
H.B.-Old(F) & $\dagger$ & & \\
R.C.-F & $*$ & & \\
R.C.-W & & $\dagger$ & $*$ \\
A.S.-F & & & $*$ \\
J.W.-F & $*$ & & \\
\hline
\end{tabular}

Note-Trends were tested by successively adding higher order components to the fitted model. A trend was tested when it was the highest order component. For example, significance of a quadratic trend means that the quadratic component of variation was significant in the fitted model PRB $=f($ ISI, ISL2); similarly, significance of a quartic trend means that the quartic trend was significant in the fitted model PRB = f(ISI, ISI2, ISI3, ISI4). ${ }^{*} p<.05 . \quad \dagger p<.01$. termed an oscillator signature (see Figure 2 and the accompanying discussion). Second, the pattern of each subject's results was reliable, as indicated by the pattern of intercorrelations given in Table 4. Replications of the finger condition by C.C. and H.B. gave very similar patterns within subjects. More importantly, the wrist and finger conditions gave very similar patterns for each of the 3 subjects. This similarity suggests that a subject's characteristic oscillator signature does not result from the biomechanical properties of particular joints or limbs. Successive replications within subjects were separated by several weeks in all cases, and different random orders of ISI conditions were used in each replication. Thus, neither similar times of testing nor orders of conditions can account for the similarity of the oscillator signatures. Third, there were differences in the patterns of bias between subjects. Although all 3 subjects produced residual bias functions that look roughly $\mathrm{W}$-shaped when plotted as in Figure 4, the individual Ws did not have exactly the same shape or location. Table 4 shows that Subjects H.B. and R.C. had similar signatures, whereas that of Subject C.C. differed from both. These similarities and differences can also be seen in Figure 4. No interpretations of the between-subject comparisons will be attempted here.

One common feature of the oscillator signatures obtained from continuation data is positive residual bias at short ISIs. It might be hypothesized that subjects cannot tap faster than some limiting rate and that this positive bias therefore reflects an inability to execute motor responses quickly enough. However, the absence of bias in synchronization data at these rates argues against a rigid limit. Since subjects can tap accurately at short ISIs when a pacer stimulus is present, the ISIs themselves must be greater than the limiting rate. Another concern is that a local nonlinearity at short ISIs might be the only real nonlinearity present. We would then expect no trends higher than quadratic in PRB functions; however, cubic and quartic trends were found in 3 subjects' data, and visual inspection of the other 2 subjects' functions suggests small, but similar, higher order trends.

The demonstration of oscillator signatures supports the hypothesis of an underlying discreteness in timing. It appears that oscillator signatures are stable over time and across biomechanically different conditions and may have somewhat different shapes for different subjects.

We next discuss an approach to measuring the shape of a subject's characteristic oscillator signature. This approach provides both an unambiguous way of locating zero crossings of the function and a summary comparison of continuation signatures with the corresponding data from synchronization tapping.

\section{Filtered PRB Functions}

One way to more clearly visualize the oscillator pattern of an individual subject is to apply a statistical filter to the PRB function. Tukey (1977) has provided methods for smoothing sequences of values to reveal nonlinear pat- 

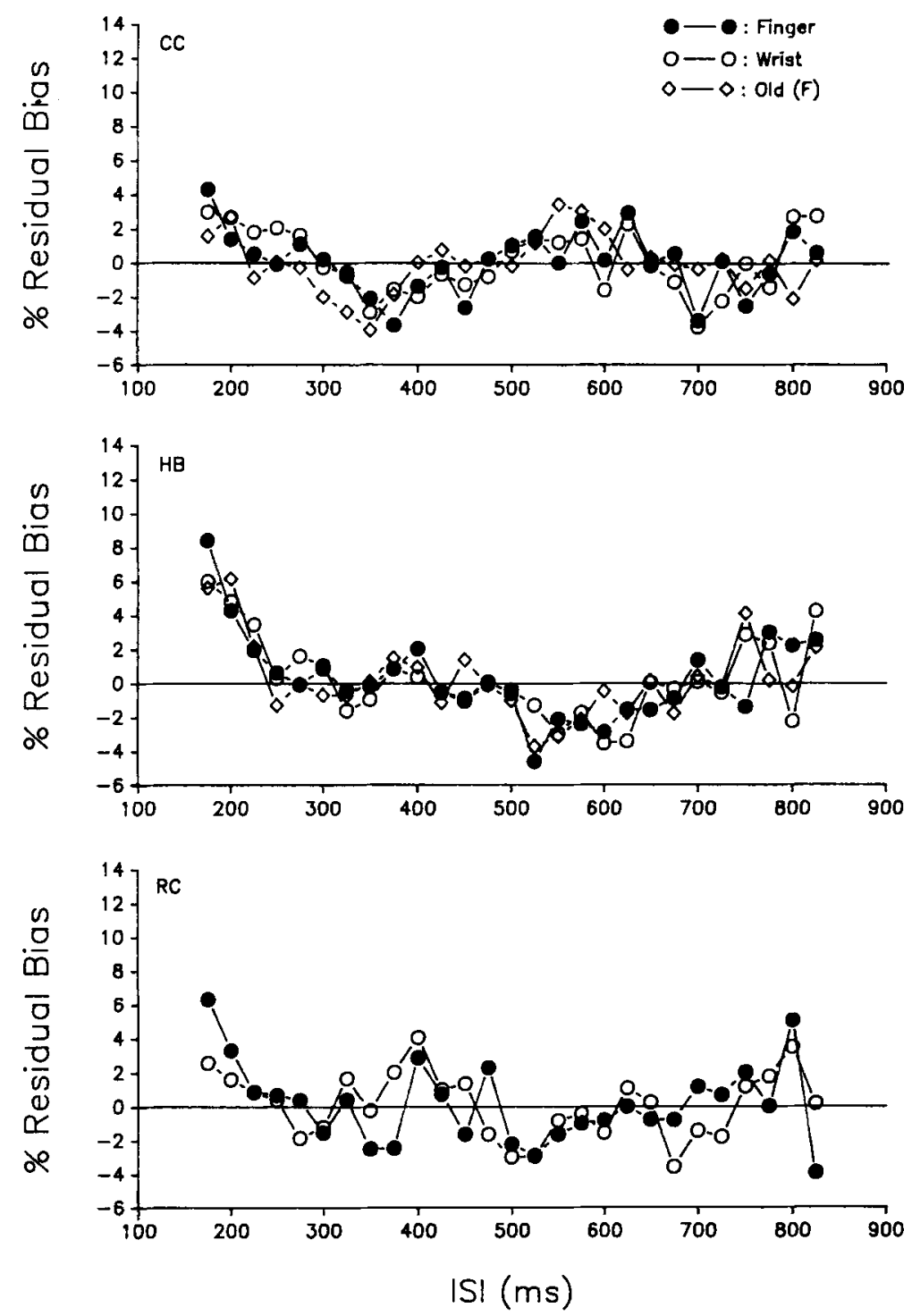

Figure 4. Percent residual bias (PRB) as a function of interstimulus interval (ISI) for each replication by Subjects C.C., H.B., and R.C. Two earlier replications by Subjects C.C. and H.B. (denoted Old[F]) are included. The similarity of a subject's replications establishes the reliability of individual oscillator signatures. The similarity of the finger and wrist conditions indicates that this level of biomechanical manipulation does not disturb a subject's oscillator signature.

Table 4

Intercorrelations of Percent Residual Bias Functions of Subjects C.C., H.B., and R.C.

\begin{tabular}{lrrrrrrr}
\hline & C.C.-F & C.C.-W & $\begin{array}{c}\text { C.C. } \\
\text { Old(F) }\end{array}$ & H.B.-F & H.B.-W & $\begin{array}{c}\text { H.B.- } \\
\text { Old(F) }\end{array}$ & R.C.-F \\
\hline C.C.-W & $.752 \dagger$ & & & & & & \\
C.C.-Old(F) & $.439 \dagger$ & $.321^{*}$ & & & & & \\
H.B.-F & .190 & .238 & -.053 & & & & \\
H.B.-W & .087 & .292 & .021 & $.757 \dagger$ & & & \\
H.B.-Old(F) & -.054 & .162 & -.040 & $.736 \dagger$ & $.806 \dagger$ & & \\
R.C.-F & .300 & .221 & .073 & $.614 \dagger$ & $.322^{*}$ & $.510 \dagger$ & \\
R.C.-W & -.078 & .168 & -.179 & $.541 \dagger$ & .261 & $.507 \dagger$ & $.519 \dagger$ \\
\hline
\end{tabular}

${ }^{*} p<.05 . \quad \dagger p<.01$. 


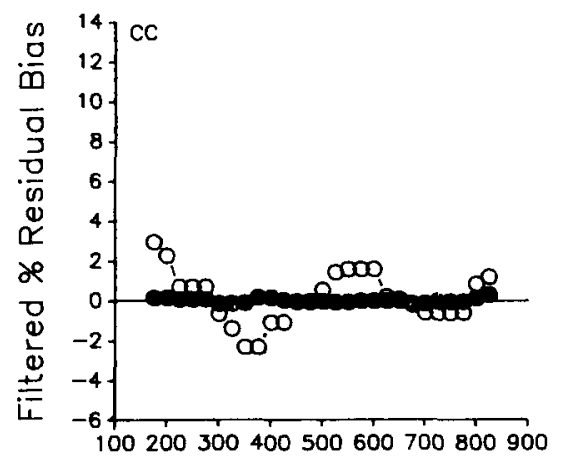

$\mid \mathrm{SI}(\mathrm{ms})$

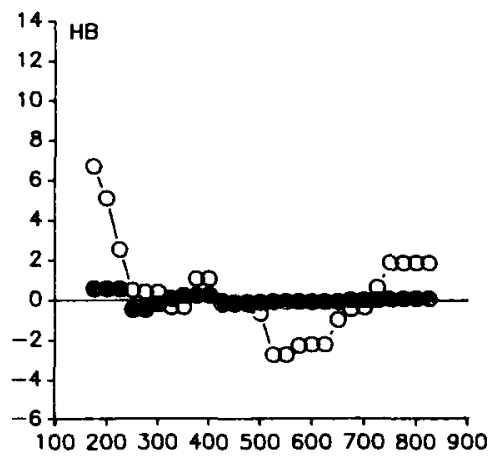

ISI (ms)

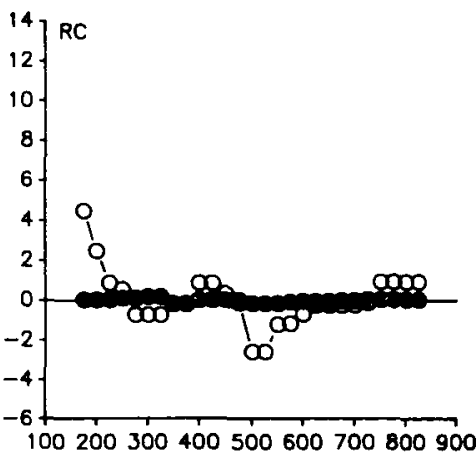

ISI (ms)
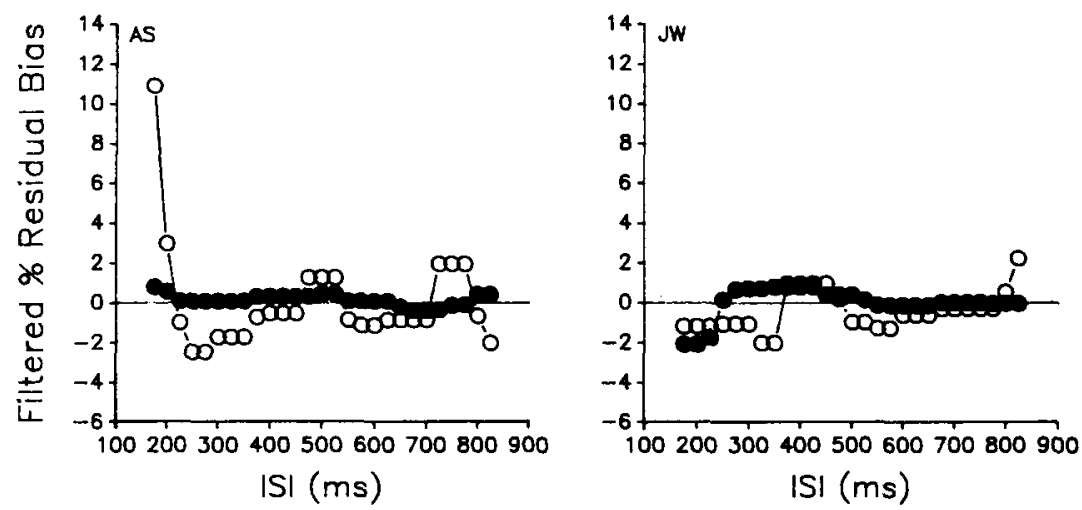

Figure 5. Synchronization and continuation: Averaged percent residual bias functions of 5 subjects, after smoothing by Tukey's (1977) 3R procedure. Oscillator signatures are apparent in continuation but are generally absent in synchronization. Tukey's procedure provides a way of clarifying the form of a series without assuming a specific function form.

terns, which do not require assuming a specific function form. This approach seemed particularly useful as a way to extract the basic form of a subject's oscillator signature.

For each subject, PRB values from all available replications of the experiment (three each for C.C. and H.B., two for R.C., and one each for 2 additional subjects, A.S. and J.W.) were averaged. Each subject's average function was then smoothed by using Tukey's (1977, pp. 210-223) 3R procedure (i.e., repeatedly taking running medians of 3 until the function exhibits no further change). Tukey's procedure for smoothing the ends of the function was also applied. Figure 5 shows the filtered functions, which we take as approximations to each subject's characteristic oscillator signature. In each panel, closed circles show synchronization data, and open circles show continuation data. In each case, the continuation function is the more oscillatory one; the synchronization function is flatter.

The filtered bias functions of Figure 5 provide a way of measuring critical time intervals such as the $\mathrm{Cl}$ and $\mathrm{C} 2$ intervals of Figure 2. When such a function is scanned from left to right, critical intervals correspond to negativegoing zero crossings of the oscillator signature. A negativegoing zero crossing can be viewed as a critical point that divides a region of positive residual bias (to its left) from a region of negative bias (to its right). A positive-going zero crossing can be viewed as a point at which there is cancellation of the opposing biases associated with the critical points on either side. The ISI values of both directions of zero crossing for each subject are given in Table 5.

\section{Variability of IRI}

Regression was also used to examine the variability of IRI, as measured by the mean of the five trial semiinterquartile ranges (SIQR) at each ISI. This measure,

Table 5

Location (in msec) of Zero Crossings of Filtered Residual Bias Functions

\begin{tabular}{lccccc}
\hline & \multicolumn{2}{c}{ First } & & \multicolumn{2}{c}{ Second } \\
\cline { 2 - 3 } \cline { 5 - 6 } Subject & Negative & Positive & & Negative & Positive \\
\hline C.C. & 287.5 & 487.5 & & 662.5 & 787.5 \\
H.B. & 312.5 & 362.5 & & 412.5 & 712.5 \\
R.C. & 262.5 & 387.5 & & 462.5 & 737.5 \\
A.S. & 212.5 & 462.5 & & 537.5 & 712.5 \\
J.W. & $175 \dagger$ & 362.5 & & 487.5 & 787.5 \\
Mean & 250 & 413 & & 513 & 748 \\
SD & 56 & 58 & & 95 & 38 \\
\hline
\end{tabular}

* Subject A.S. had a third negative crossing at $788 \mathrm{msec}$. IIn the raw data, there was a positive bias at $175 \mathrm{msec}$ for all 5 subjects, changing to negative bias at differing larger ISIs. The filtering algorithm removed the original bias value of $+12.4 \%$ at $175 \mathrm{msec}$ for Subject J.W., since it was a single extreme value. Thus, assignment of first zero crossing at $175 \mathrm{msec}$ for J.W. was deemed a reasonable estimate. 
which is proportional to the standard deviation for normally distributed data $(\mathrm{SIQR}=.67 \times S D)$, was approximately a linear, function; of ISI, and so exhibited the familiar scalar property found with many types of timing and time-perception data (e.g., Gibbon et al., 1984). We referred earlier to this linearity in explaining why the percentage transformation was appropriate. Table 2 gives the parameters of the linear fits for each set of data.

Table 2 also gives the regression parameters for the square of semi-interquartile range, which under normality is proportional to variance $\left(\mathrm{SIQR}^{2}=.45 \times\right.$ variance $)$, as a function of ISI. The goodness-of-fit measure $r^{2}$ indicates that SIQR gives better linearity. Furthermore, the intercepts of linear fits to the SIQR ${ }^{2}$ values are often negative; $\operatorname{SIQR}^{2}$ cannot have negative values because it is a squared measure, so at small ISIs it must violate a simple linear model. In sum, the variability measures that bear linear relations to ISI are SIQR and standard deviation, not $\mathrm{SIQR}^{2}$ and variance.

Residual variability was defined as the difference between the semi-interquartile range and the linear predictions based on the fits of Table 2. We sought to determine if there was a relationship between the oscillator signatures of the PRB data and the corresponding values of residual variability. Two relationships were thought to be possible. Under the first, which we term the identification pattern, residual variability would exhibit a local minimum near each of the negative-going zero crossings (critical intervals) given in Table 2 . The rationale for the identification pattern is that if the critical intervals are "preferred" times, then they may be represented in the timing system with relatively low variance. Under the second possible relationship, which we term the discrimination pattern, residual variability would exhibit a local minimum near the positive-going zero crossings (category boundaries). The rationale for the discrimination pattern is that if the critical intervals are in the middle of temporal "categories," then differential timing control may be better near a category boundary than in the middle of the category.

Residual bias was cross-correlated with residual variability over 15 lags centered on $\mathbf{0}$. (Zero lag corresponds to a traditional correlation of the two measures. A lag of -1 would shift one series relative to the other such that, for example, the residual bias at ISI $=500 \mathrm{msec}$ would be paired with the residual variability at ISI $=475 \mathrm{msec}$. Recall that ISI was manipulated in steps of $25 \mathrm{msec}$.) The logic of the cross-correlation analysis was as follows: If residual variability, like residual bias, alternately increases and decreases over ISI, then a certain phase relationship will hold between the two series. A shift of one sequence of values relative to the other will bring them closest to congruence with each other and so maximize their crosscorrelation. In the identification pattern, high variability is associated with negative-going zero crossings of the oscillator signature, so the shift predicted to maximize the cross-correlation will be a leftward shift of the variability sequence relative to the bias sequence. Conversely, the dis- crimination pattern predicts that a rightward shift will have this effect. However, the cross-correlation patterns were not consistent, either within subjects or between subjects. Of the eight more recent replications, five had no statistically significant correlations $(p>.05)$ between the two series at any lag. The three replications with at least one significant correlation had them located at different lags. None of the cross-correlation analyses revealed the discrimination pattern; two conformed to the identification pattern. However, neither the identification nor the discrimination pattern can be said to have received strong confirmation from these data, and we cannot reject the simpler hypothesis of independence between residual bias and residual variability.

\section{Summary of Results}

This experiment demonstrated small but reliable nonlinearities in the production of time intervals by manual tapping. The nonlinearities were of the sort predicted under a categorical conception of motor timing; we have applied the term oscillator signature to these effects. There are individual differences in the form of the oscillator signature. However, an individual subject's signature seems to be invariant over at least two biomechanically different types of responding: finger flexion and wrist flexion. The oscillator signature is a characteristic of continuation, in which the subject maintains a tapping rate from memory, but not synchronization, in which a pacer stimulus is available to guide corrections of timing.

\section{GENERAL DISCUSSION}

Two levels of description are needed to give an account of the timing performance observed in this experiment. At a relatively gross level, we found that the subjects' tapping rate approximately matched the stimulus rate of each ISI condition. Thus, the identity function, or a line differing from it only slightly, provided a good overall description of each set of data. If the residuals around these functions had consisted of unsystematic variation, we would at this point opt for a continuous conception of timing. However, residual bias was systematically related to ISI-and in the way predicted by a categorical conception of timing.

The evidence for categorical time production came from residual bias data from the continuation part of our experimental trials. We expected that continuation would provide the best opportunity to see the effects of an endogenous timing mechanism, whereas synchronization performance must reflect a combination of endogenous and exogenous timing factors, with the exogenous tending to cancel endogenous categorical effects, as well as unsystematic sources of bias.

We derive the following picture of the timing system: There appear to be oscillatory processes, with stable and measurable rates, whose influence on time-interval production can be seen in the patterns of residual bias that we have examined here. The motor system, and perhaps 
also some perceptual processes, may use these oscillators as temporal references. We think that, at present, this view is better supported than an alternative one based on biomechanics, because oscillator signatures obtained with finger and with wrist flexion were equivalent. The traces of the oscillators are small; residual bias, even at its most extreme, seems to be only about $4 \%$ of the produced interval. However, this small value, together with the apparent existence of multiple oscillators with different intrinsic rates, means that the timing system as a whole can come very close to the performance of a continuously adjustable oscillator. One possibility is that a neural mechanism provides discrete oscillatory reference values that are subject to considerable, but still somewhat constrained, entrainment. Another possibility is that the oscillatory rates are relatively fixed and that the contribution of each to performance depends on its proximity to ISI. Finally, the question of whether motor timing is the main function of these hypothetical oscillatory processes or is an emergent property cannot be answered here. The present experiment cannot rule out the possibility that oscillator signatures are caused by interference with an otherwise continuously adjustable timing mechanism from other oscillatory processes. The view that categorical time production is based on a categorical timing mechanism is potentially related to other theoretical proposals, of which we will mention two.

\section{Relationship to a Two-Process Model of Timing}

Wing and Kristofferson (1973a, 1973b) proposed a twoprocess model of the timing of repetitive acts such as tapping. The two processes are a timekeeper (or a clock) and a motor system capable of generating discrete responses. This model provides a good account of a variety of data collected by Wing and others. In particular, the model can be used to analyze the variability of IRIs into components associated with the timekeeper and the motor system. Essentially the same model was proposed independently by Pittendrigh and Daan (1976) to separate variability in a circadian clock from variability in the time to begin circadian activity once the correct clock time is reached. Wing (1973) found that timekeeper variance increased with ISI over the domain 220 to $490 \mathrm{msec}$, whereas motor variance remained constant.

Our finding regarding oscillator signatures can be seen as revealing a property of the timekeeper in Wing's model. If Wing's timekeeper is the output of an entrained oscillator, it is subject to positive bias at some rates and negative bias at others. The residual bias patterns we have described here could be interpreted as a description of the limits of entrainment of the oscillators underlying Wing's timekeeper.

\section{Relationship to Duration-Discrimination Findings}

Kristofferson (1980, 1984), interpreting an extensive series of experiments on duration discrimination, proposed temporal categories or steps to account for the interactive effects of base duration and practice on the variability of judgments. Kristofferson's categories follow a doubling rule such that (after extensive practice) his quantal variability measure $q$ roughly doubled from 12 to 25 to 50 to 100 as base duration correspondingly doubled at 200 , 400 , and $800 \mathrm{msec}$. Between the doubling points, $q$ was relatively insensitive to base duration. (The measure $q$ in Kristofferson's theory is the unit of psychological time and thus determines temporal resolution in a durationdiscrimination task.)

Kristofferson's depiction of duration discrimination across different base durations roughly parallels our own depiction of time production across different ISIs. Both are two-level descriptions of performance, allowing for approximate continuity overall, but with a fine-grain analysis providing evidence for underlying discreteness. The parallel is suggestive, although not yet complete. It will be important in future work to ask whether both time production and time perception reveal the same oscillators.

\section{Is Timing Smooth or Discrete?}

The answer to this question depends on the level of description and analysis. At present, psychological time seems to possess aspects of both smoothness and discreteness. The subjective smoothness of time is certainly not contradicted by the present findings, and, in fact, is impressively corroborated by the striking linearity of the functions relating IRI to ISI. However, the small systematic inaccuracies around these linear functions, which form the patterns we have called oscillator signatures, provide evidence for an underlying discreteness in motor timing. We therefore suggest that, although well-disguised, the fundamental property of the mechanism for this type of motor timing is a set of discrete intrinsic time intervals that serve as temporal reference values.

\section{REFERENCES}

Bartlett, N., BARTlett, S. (1959). Synchronization of a motor response with an anticipated sensory event. Psychological Review, 66, 203-218.

Church, R. M., GetTy, D. J., \& Lerner, M. D. (1976). Duration discrimination by rats. Joumal of Experimental Psychology: Animal Behavior Processes, 2, 303-312.

Collyer, C. E., Broadbent, H. A., \& Church, R. M. (1990, June). Preferred rates of repetitive tapping are not strongly determined by physical dynamics. Paper presented at the convention of the American Psychological Society, Dallas.

Creelman, C. D. (1962). Human discrimination of auditory duration. Journal of the Acoustical Society of America, 34, 582-593.

DUNLAP, K. (1910). Reactions to rhythmic stimuli, with attempt to synchronize. Psychological Review, 17, 399-416.

FraneK, M., RAdil, T., IndRa, M., \& LANSKY, P. (1987). Following complex acoustical patterns by tapping. Insemational Joumal of Psychophysiology, 5, 187-192.

GibBoN, J. (1977). Scalar expectancy theory and Weber's Law in animal timing. Psychological Review, 84, 279-325.

Gibbon, J. (1991). Origins of scalar timing. Learning \& Motivation, 22, 3-38.

Gibbon, J., Сhurch, R. M., Meck, W. H. (1984). Scalar timing in memory. In J. Gibbon \& $\mathrm{L}$. Allan (Eds.), Timing and time perception (Annals of the New York Academy of Sciences, Vol. 423, pp. 52-77). New York: New York Academy of Sciences. 
Glass, L., Mackey, A. (1988). From clocks to chaos. San Francisco: W. H. Freeman.

Harnad, S. (Ed.) (1987). Categprical perception. Cambridge: Cambridge University Press.

HARY, D., \& MOORE, G. P. (1985). Temporal tracking and synchronization strategies. Human Neurobiology, 4, 73-77.

HARY, D., Moore, G. P. (1987). Synchronizing human movement with an external clock source. Biological Cybermetics, 56, 305-311.

JAMES, W. (1950). The principles of psychology. New York: Dover. (Original work published 1890)

Kristofferson, A. B. (1980). A quantal step function in duration discrimination. Perception \& Psychophysics, 27, 300-306.

Krustofferson, A. B. (1984). Quantal and deterministic timing in human duration discrimination. In J. Gibbon \& L. Allan (Eds.), Timing and time perception (Annals of the New York Academy of Sciences, Vol. 423, pp. 3-15). New York: New York Academy of Sciences.

Matin, E. (1974). Saccadic suppression: A review and an analysis. Psychological Bulletin, 81, 899-917.

NAGASAK, H. \& NAGASAKI, N. (1982). Rhythm formation and its disturbances: A study based on periodic response of a motor output system. Journal of Human Ergology, 11, 127-142.

Peters, M. (1989). The relationship between variability of intertap intervals and interval duration. Psychological Research, 51, 38-42.

Pittendrigh, C. S., DAAN, S. (1976). A functional analysis of circadian pacemakers in nocturnal rodents: $I$. The stability and lability of spontaneous frequency. Journal of Comparative Physiology, 106, 223-252.
Rosenbaum, D. A., Slotta, J. D., Vaughan, J., a Plamondon, R. J. (1991). Optimal movement selection. Psychological Science, 2, 86-91. Stevens, L. T. (1886). On the time sense. Mind, 11, 393-404.

TUkEY, J. W. (1977). Exploratory data analysis. Reading, MA: Addison-Wesley.

WING, A. M. (1973). The timing of interresponse intervals by human subjects. Unpublished doctoral dissertation, McMaster University, Hamilton, Ontario.

WING, A. M. (1977). Effects of type of movement on the temporal precision of response sequences. British Journal of Mathematical \& Statistical Psychology, 30, 60-72.

WiNG, A. M. (1980). The long and short of timing in response sequences. In G. E. Stelmach \& J. Requin (Eds.), Tutorials in motor behavior. Amsterdam: North-Holland.

Wing, A. M., Сhurch, R. M., Gentner, D. (1989). Variability in the timing of responses during repetitive tapping with alternate hands. Psychological Research, 51, 28-37.

Wing, A. M., KRIstofferson, A. B. (1973a). Response delays and the timing of discrete motor responses. Perception \& Psychophysics, 14, 5-12.

Wing, A. M., Kristofferson, A. B. (1973b). The timing of interresponse intervals. Perception \& Psychophysics, 13, 455-460.

Woodrow, H. (1932). The effect of rate of sequence upon the accuracy of synchronization. Journal of Experimental Psychology, 15, 357-379.

(Manuscript received December 26, 1990; revision accepted for publication September 12, 1991.) 